\title{
INVESTIGACIÓN
}

\section{Estudio cinético del proceso de digestión anaerobia de aguas de lavado de aceitunas de almazara en reactores de mezcla completa con microorganismos inmovilizados}

\author{
Por Rafael Borja (1), José Alba (1), Antonio Martín (2) y Angela Mancha (1) \\ (1) Instituto de la Grasa (C.S.I.C.). Avda. Padre García Tejero, 4. 41012 - Sevilla.
}

(2) Departamento de Ingeniería Química. Facultad de Ciencias. Universidad de Córdoba.

Avda. San Alberto Magno, s/n. 14004 - Córdoba.

\section{RESUMEN}

Estudio cinético del proceso de digestión anaerobia de aguas de lavado de aceitunas de almazara en reactores de mezcla completa con microorganismos inmovilizados.

Se ha efectuado un estudio cinético del proceso de digestión anaerobia en régimen continuo de aguas de lavado de aceitunas de almazara en un reactor de mezcla completa utilizando sepiolita como soporte para la inmovilización de los microorganismos responsables del proceso, a la temperatura de $35^{\circ} \mathrm{C}$.

Se utilizan cuatro concentraciones de sustrato como alimento $4,5,3,5,2,5$ y $1,5 \mathrm{~g} \mathrm{DQO} / \mathrm{l}$, operando con cada una de ellas en un rango de tiempos de retención hidráulicos de 4,5 a 1,25 días.

Para ello, se aplica el método cinético de Michaelis-Menten, obteniéndose los valores de la velocidad máxima de utilización de sustrato, $k$ (g DQO/g SSV día), y de la constante cinética del proceso, $K_{\mathrm{s}}(\mathrm{g} \mathrm{DQO} /$ ), para cada concentración de sustrato objeto de estudio. Se observa que estas constantes cinéticas disminuyen de forma significativa al disminuir la concentración de sustrato en el alimento, presentando los valores más elevados para la mayor concentración utilizada.

Los coeficientes de producción celular, $Y_{x}$ y de muerte microbiana, $k_{\mathrm{d}}$, fueron $0,14 \mathrm{~g} \mathrm{SSV} / \mathrm{g}$ DQO y $0,003 \mathrm{dias}^{-1}$, respectivamente.

PALABRAS-CLAVE: Aceituna - Aguas de lavado - Cinética Digestión anaerobia - Microorganismos inmovilizados - Reactor de mezcla completa.

\section{SUMMARY}

Kinetic study of anaerobic digestion of wastewaters from the washing of olives prior to the oil production process in a completely mixed reactor with immobilised microorganisms.

A kinetic study of the continuous anaerobic digestion process of wastewaters from the washing of olives prior to the oil production process was carried out in a completely mixed reactor with sepiolite immobilised biomass at $35^{\circ} \mathrm{C}$.
Four influent COD concentrations, 4,5, 3,5, 2,5 y 1,5 g COD/ were used as substrate operating for a hydraulic retention time range of 4,5 to 1,25 days.

The Michaelis-Menten model has been applied, obtaining the values of the maximum substrate utilization rate, $k(\mathrm{~g} \mathrm{COD} / \mathrm{g}$ VSS day), and the kinetic constant of the process, $K_{\mathrm{s}}$ (g COD/l), for each influent substrate concentration studied. The values of these parameters significantly decreased with decreasing concentration of influent wastewater, showing the highest values for the greatest influent substrate concentration used as feed.

The microbial yield coefficient, $Y_{X}$, and sludge decay rate coefficient, $k_{\mathrm{d}}$, were also determined to be $0,14 \mathrm{~g} \mathrm{VSS} / \mathrm{g}$ COD and 0,003 days $^{-1}$, respectively.

KEY-WORDS: Anaerobic digestion - Completely mixed reactor - Immobilised microorganisms - Kinetic - Olive - Waste water from washing.

\section{INTRODUCCIÓN}

La instalación de máquinas lavadoras de aceitunas se ha generalizado en las almazaras, fundamentalmente para disminuir los costos individuales de eliminación de impurezas.

La aplicación de los nuevos sistemas de recolección ha aumentado el rendimiento de esta operación $\mathrm{y}$ ha introducido en algunos casos nuevos criterios $\mathrm{y}$ equipos para la recogida de las aceitunas, como son las barredoras, sopladoras y aspiradoras. Todo esto conduce a que las almazaras tienen que disponer de equipos de limpieza y lavado de gran capacidad para poder eliminar todas las impurezas de las aceitunas que en muchos casos llegan a superar el $30-40 \%$, sobre todo cuando por alguna causa meteorológica aumenta el nivel de aceitunas de suelo.

La utilización de estos equipos de lavado de aceitunas origina unos niveles de producción de aguas de lavado comprendidos entre 0,05-0,09 $1 / \mathrm{kg}$ de aceitunas (Alba, 1997). Teniendo en cuenta que la 
capacidad media de procesado de las almazaras de Andalucía suele ser de 150 t aceitunas/día, cada instalación de las aproximadamente 800 existentes, produce entre 7.500-13.500 I de aguas de lavado/día. La carga contaminante de estas aguas residuales, de grado bajo-medio, no hace aconsejable su vertido a los cauces públicos y determina la necesidad de un tratamiento previo de depuración, utilizando alguno de los sistemas y tecnologías existentes.

Estudios preliminares pusieron de manifiesto la viabilidad de la utilización de procesos de depuración biológica para el tratamiento de estos efluentes. En este sentido, procesos clásicos aerobios de fangos activados sin recirculación de lodos permitieron la degradación del $67 \%$ de la demanda química de oxígeno (DQO) de este residuo operando con 6 horas de tiempo de retención hidráulico (TRH) a la temperatura de $14^{\circ} \mathrm{C}$, incrementándose considerablemente la velocidad de utilización de sustrato cuando se trabaja a $28^{\circ} \mathrm{C}$ (Borja et al., 1995a). Sin embargo, aunque los procesos clásicos aerobios han demostrado su aplicabilidad práctica para el tratamiento biológico de estas aguas residuales, los procesos anaerobios presentan claras ventajas en relación a los anteriores, entre las que se pueden destacar las siguientes (Olthof and Oleszkiewick, 1982):

- Las necesidades energéticas son del orden de la décima parte de los aerobios, ya que se suprime el consumo correspondiente a la aireación.

- Las bacterias anaerobias transforman la sustancia orgánica en gas metano.

- Los problemas en la eliminación de lodos se reducen en un $80-85 \%$.

- Escaso requerimiento de nutrientes.

- Se evitan malos olores al desarrollarse en sistemas cerrados.

De esta forma, en estudios de digestión anaerobia realizados en reactores discontinuos con biomasa adherida se observaron eficiencias de eliminación de DQO superiores al $64 \%$, con coeficientes de rendimiento en metano variables entre 0,263 y 0,298 $\mathrm{ICH}_{4} / \mathrm{g} \mathrm{DQO}$, dependiendo del grado de maduración de la aceituna (Borja et al., 1994).

En un trabajo precedente (Borja et al., 1998) se estudió el proceso de digestión anaerobia de estos efluentes en régimen continuo utilizando un reactor de lecho fluidizado con sepiolita como soporte de adhesión bacteriana a la temperatura de $35^{\circ} \mathrm{C}$. El reactor operó de modo satisfactorio utilizando concentraciones de alimento de 4,5,3,5,2,5 y 1,5 g DQO/I en un rango de 4,5 a 1,25 días de tiempo de retención hidráulico. De esta forma, se obtuvieron eficiencias de eliminación de DQO entre el 50 y el $90 \%$ para velocidades de carga orgánica variables entre 0,46 y 2,25 g DQO/l día, utilizando la concentración de sustrato más elevada $\left(S_{0}=4,5 \mathrm{~g} D Q / / \mathrm{l}\right)$. La DQO y acidez volátil de los efluentes se incrementaron de forma paralela al aumentar la velocidad de carga orgánica en el reactor, presentando esta última un valor máximo de $1,55 \mathrm{~g}$ ácido acético/l en el caso más desfavorable estudiado ( $\mathrm{S}_{0}=4,5 \mathrm{~g}$ DQO/l; $\mathrm{TRH}=1,25$ días). Sin embargo, las subidas de acidez volátil fueron contrarrestadas en todo momento por los elevados valores de alcalinidad de los efluentes del reactor lo que dio lugar a una gran estabilidad del sistema, como se demostró por los adecuados valores de $\mathrm{pH}$ alcanzados $(6,7-7,6)$ y los pequeños valores de la relación acidez volátil/alcalinidad observados en todo momento, en la mayoría de los casos, muy por debajo del límite recomendado $(0,3-0,4)$ por encima del cual el proceso anaerobio se desestabiliza.

Una vez verificada la aplicabilidad, eficiencia y estabilidad del proceso de digestión anaerobia de estas aguas residuales (Borja et al., 1998), el objetivo de este trabajo, como complemento y ampliación del estudio anterior, es el de obtener las constantes cinéticas que rigen este proceso utilizando un reactor de mezcla completa con microorganismos inmovilizados. Para ello, se ha utilizado el modelo cinético de Michaelis-Menten que permite obtener la velocidad máxima de utilización de sustrato y la constante cinética del proceso de depuración anaerobia de estas aguas residuales. Paralelamente se ha determinado el coeficiente de muerte celular, así como el coeficiente de producción microbiana.

\section{PARTE EXPERIMENTAL}

\subsection{Equipo experimental}

El reactor utilizado está constituido por un cilindro de vidrio de $6 \mathrm{~cm}$ de diámetro interno y $40 \mathrm{~cm}$ de altura, con un volumen efectivo de 1 litro, al que se acopla una pieza troncocónica en la parte superior para facilitar la decantación de la biomasa, con el fin de evitar su pérdida con el efluente del proceso. Todo este equipo se introduce en una cámara termostatizada a la temperatura de $35^{\circ} \mathrm{C}$. Como soporte para la adhesión de los microorganismos responsables del proceso se utiliza sepiolita, de tamaño comprendido entre 0,4 y $0,8 \mathrm{~mm}$, que permite la adhesión preferencial de bacterias metanogénicas (Maestrojuán y Fiestas, 1988; Fiestas y Borja, 1996) en relación a las hidrolíticas y acetogénicas. Ello da lugar a un buen comportamiento de este soporte desde el punto de vista cinético. Por otra parte, presenta una pequeña densidad aparente $(70 \mathrm{~g} / \mathrm{l})$, lo que le hace idóneo para el desarrollo de procesos de depuración en este tipo de reactores.

La agitación y fluidización de la biomasa bacteriana se realiza con un sistema de agitación magnética (365 revoluciones por minuto). La alimentación se realiza por la parte inferior del reactor mediante una 
bomba peristáltica conectada a un temporizador que permite la adición de los distintos caudales de influente en varias dosis diarias. La salida del efluente se realiza por la parte superior del decantador a través de un cierre hidráulico. Otros detalles del equipo experimental relativos al procedimiento seguido para la determinación del volumen de metano producido en función del tiempo se detallan en un trabajo precedente (Borja et al., 1991).

\subsection{Características de las aguas de lavado de aceitunas}

Las aguas de lavado de aceitunas (variedad picual) utilizadas en esta serie de experimentos proceden de las instalaciones de lavado existentes en la Almazara Experimental del Instituto de la Grasa. El efluente base utilizado presentó un valor de DQO de $4,5 \mathrm{~g} / \mathrm{l}$. El pH, la acidez volátil y alcalinidad del mismo fueron 5,9, 0,62 $\mathrm{g} / \mathrm{l}$ (ácido acético) y 0,48 $\mathrm{g} / \mathrm{l}\left(\mathrm{CaCO}_{3}\right)$ respectivamente. Las características completas relativas al contenido en nitrógeno amoniacal, fenoles totales, sólidos totales y en suspensión, minerales y volátiles, del agua residual objeto de estudio se encuentran resumidas en un trabajo precedente (Borja et al., 1998). Los otros tres efluentes residuales utilizados como sustrato, de concentración en DQO, $3,5,2,5$ y $1,5 \mathrm{~g} / \mathrm{l}$, se obtuvieron por dilución con agua destilada del agua residual de partida anteriormente mencionada.

Las características de las aguas de lavado así como de los efluentes resultantes del proceso de digestión anaerobia se determinaron de acuerdo con el «Standard Methods for the Examination of Water and Wastewater" (American Public Health Association, 1985).

\subsection{Procedimiento experimental}

Tras las etapas de inoculación y aclimatación de la biomasa utilizada como inóculo al sustrato objeto de estudio, que quedaron expuestas en un trabajo precedente (Borja et al., 1994), se efectuaron una serie de experimentos en régimen discontinuo en los que se procesaron aguas de lavado con valores de DQO variables entre 2,8 y $5,5 \mathrm{~g} / \mathrm{l}$, correspondientes a aceitunas con distinto grado de maduración pertenecientes a la campaña oleícola 1992-93.

Finalizadas estas etapas preliminares se efectuaron cuatro series de experimentos en régimen continuo, en los que se trataron aguas de lavado de aceitunas de 4,5 g DQO/l (agua residual original obtenida en el proceso industrial de lavado), 3,5, 2,5 y $1,5 \mathrm{~g} \mathrm{DQO} / \mathrm{l}$, éstas tres últimas obtenidas por dilución de la primera. Para cada uno de estos cuatro influentes, el reactor operó con caudales de alimentación variables entre 0,22 y $0,80 \mathrm{l} /$ día, que se corresponden con tiempos de retención hidráulicos de 4,5, 3,5, $2,5,2,0,1,5$ y 1,25 días. Para el cálculo de estos tiempos de retención se ha considerado únicamente el volumen efectivo del reactor (1 litro) sin tener en cuenta el volumen del decantador troncocónico, ya que la concentración de biomasa observada en este último es totalmente despreciable (Tabla I) en relación a la concentración de biomasa activa existente en el volumen mencionado de reactor $(11,2,11,4$ 11,5 y 11,7 g sólidos en suspensión volátiles, SSV/I como valores medios para los experimentos con concentraciones de sustrato en el alimento de 4,5, $3,5,2,5$ y $1,5 \mathrm{~g} \mathrm{DQO} / \mathrm{l}$, respectivamente).

\section{Tabla I}

Concentración de sólidos en suspensión volátiles en los efluentes, $X_{e}$ (g SSV/I), en función del tiempo de retención hidráulico $(\mathrm{TRH})$ para las distintas concentraciones de sustrato $\left(S_{0}\right)$ utilizadas

\begin{tabular}{ccccc}
\hline $\begin{array}{c}\text { TRH } \\
\text { (dias) }\end{array}$ & $s_{0}=4,5 \mathrm{gDQON}$ & $s_{0}=3,5 \mathrm{~g}$ DQON & $s_{0}=2,5 \mathrm{~g}$ DQO/ & $s_{0}=1,5 \mathrm{~g} \mathrm{DQO}$ \\
\hline $\mathbf{4 , 5}$ & 0,352 & 0,241 & 0,119 & 0,026 \\
$\mathbf{3 , 5}$ & 0,376 & 0,251 & 0,157 & 0,053 \\
$\mathbf{2 , 5}$ & 0,375 & 0,257 & 0,175 & 0,073 \\
$\mathbf{2 , 0}$ & 0,345 & 0,258 & 0,170 & 0,077 \\
$\mathbf{1 , 5}$ & 0,297 & 0,251 & 0,143 & 0,072 \\
$\mathbf{1 , 2 5}$ & 0,267 & 0,193 & 0,124 & 0,059 \\
\hline
\end{tabular}

Para cada caudal de alimentación o tiempo de retención hidráulico correspondiente, se determinan tras alcanzarse condiciones de estado estacionario el volumen de metano producido diariamente, el pH, la DQO, acidez volátil y alcalinidad de los efluentes obtenidos. Las muestras de efluentes se tomaron y analizaron durante al menos 5 días consecutivos. Se consideran como valores correspondientes al estado estacionario las medias de estas determinaciones cuando las desviaciones entre los valores observados son iguales o inferiores al $3 \%$ (Borja et al., 1998).

Los experimentos correspondientes a cada sustrato empleado se efectúan por orden creciente de caudal de alimentación, con objeto de evitar los posibles efectos negativos resultantes de "sobrecargas" orgánicas, por aumentos bruscos en la carga alimentada.

\section{RESULTADOS Y DISCUSIÓN}

En el trabajo precedente que se menciona en apartados anteriores (Borja et al., 1998) se realizó 
una discusión detallada del comportamiento operacional del reactor, así como de la evolución con el tiempo de retención hidráulico de las variables de control más destacadas del proceso de digestión anaerobia de estas aguas residuales, como son el caudal de metano producido, DQO, acidez volátil, alcalinidad y concentración de sólidos (totales y en suspensión, minerales y volátiles) de los efluentes del proceso. En este sentido, se observó que el reactor operó de modo satisfactorio para las cuatro concentraciones de sustrato estudiadas $(4,5,3,5$, $2,5,1,5 \mathrm{~g} \mathrm{DQO} / \mathrm{l})$ en un rango de tiempos de retención hidráulicos variable entre 4,5 y 1,25 días. Así por ejemplo, para las concentraciones de sustrato de 4,5 y $3,5 \mathrm{~g} \mathrm{DQO} / \mathrm{l}$ el porcentaje de eliminación de DQO varió entre el $50-90 \%$ y el $51-89 \%$, respectivamente. Los incrementos en la acidez volátil de los efluentes se contrarrestaron en todo momento por los elevados valores de alcalinidad observados, lo que dio lugar a que la relación acidez volátil/alcalinidad se mantuviera, a excepción del experimento correspondiente al tiempo de retención más pequeño ( 1,25 días) con el alimento más concentrado $(4,5 \mathrm{~g} \mathrm{DQO} / \mathrm{l})$, por debajo del rango recomendable $(0,3-0,4)$ a partir del cual el proceso comienza a desestabilizarse (Fannin, 1987; Field et al., 1988).

Por otra parte, el $\mathrm{pH}$ del sistema se mantuvo, prácticamente, en todos los casos dentro del intervalo óptimo $(6,8-7,6)$ para el adecuado desarrollo de las bacterias metanogénicas (Fannin, 1987), mientras que el coeficiente medio de producción de metano fue de 0,281 metano STP/g DQO eliminada (Borja et al., 1998).

Para obtener las constantes cinéticas que rigen el proceso de digestión anaerobia de aguas de lavado de aceitunas se utiliza el modelo cinético de Michaelis-Menten.

Dada la existencia de mezcla completa en el reactor anaerobio un balance de sustrato (DQO) conduce a la ecuación:

$$
\mathrm{d}(V S) / \mathrm{d} t=q S_{0}-q S-U V X
$$

donde $U$ es la velocidad de consumo de sustrato por unidad de masa de microorganismos presentes en el biorreactor (g DQO/g SSV día), obtenida mediante determinaciones de la DQO de los efluentes y de las distintas concentraciones de alimento; $S_{0}$ y $S$ son las concentraciones de sustrato a la entrada y salida del biorreactor (g DQO/l); $q$ es el caudal volumétrico de la alimentación (I/día); $V$ el volumen del biorreactor (I); $t$ el tiempo (días) y $X$ la concentración de biomasa evaluada a través del contenido en sólidos en suspensión volátiles, SSV (g SSV/I).
Para el estado estacionario $\mathrm{d}(V S) / \mathrm{d} t=0$, con lo que $U$ se puede calcular por la expresión:

$$
U=\left(S_{0}-S\right) /(X \theta)
$$

donde $\theta$ es el tiempo de retención hidráulico definido por: $\theta=V / q$.

Mientras la biomasa es retenida en el biorreactor, el tiempo de retención hidráulico es relativamente pequeño comparado con el tiempo de retención celular. En estas circustancias el sistema no puede alcanzar el estado estacionario en sentido estricto desde el punto de vista de la fase sólida, pero sin embargo las condiciones de estado estacionario son aplicables tanto a la fase líquida como al sustrato soluble. Las variaciones en la concentración de biomasa durante intervalos de tiempo relativamente amplios son despreciables en relación con el contenido en biomasa del reactor, de manera que prácticamente no contribuyen a la dinámica del sistema. En tal estado "pseudoestacionario" para la resolución de los balances de materia para la fase soluble y para la sólida se admite que $\mathrm{d} S / \mathrm{d} t=0$ (no hay acumulación de sustrato) y $\mathrm{d} X / \mathrm{d} t \neq 0$, suponiendo que la velocidad volumétrica de generación de biomasa puede ser constante en el intervalo «pseudoestacionario» considerado (Guiot et al., 1989). Por tanto, en estas condiciones, la velocidad específica de consumo de sustrato puede determinarse mediante la ecuación (2).

En la figura 1 se han representado las parejas $U v s S$. La forma de las curvas sugiere que el proceso obedece a una ecuación tipo Michaelis:

$$
U=k S /\left(K_{\mathrm{s}}+S\right)
$$

lo que se confirma por la linealidad de la representación $1 / U$ vs $1 / S$ (representación de Lineweaver-Burk, figura 2) para las cuatro concentraciones de sustrato utilizadas como alimento:

$$
1 / U=1 / k+\left(K_{s} / k\right)(1 / S)
$$

donde $k$ (g DQO/g SSV día) es la velocidad máxima de utilización de sustrato y $K_{\mathrm{s}}$ (g DQO/l) es la constante cinética del proceso.

Este modelo cinético también tiene en cuenta las siguientes consideraciones (Shieh et al., 1985):

- La reacción tiene lugar en el volumen efectivo del reactor ocupado por la biomasa activa adherida.

- La ecuación de Michaelis-Menten es aplicable para describir la variación de la velocidad específica de utilización de sustrato con la concentración de sustrato en el efluente.

- La alimentación no contiene los microorganismos responsables del proceso anaerobio y la 
concentración de SSV en el efluente puede considerarse como la biomasa no adherida o desprendida del reactor, en este caso, totalmente despreciable en relación con el contenido en biomasa del mismo (tabla I).

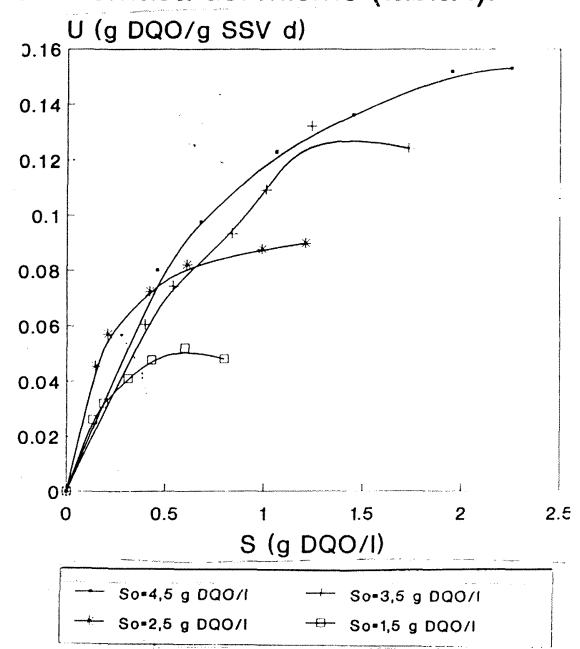

Figura 1

Variación de la velocidad específica de consumo de sustrato, $U$ (g DQO/g SSV d) con la concentración de sustrato en el efluente, $S(g \mathrm{DQO} / \mathrm{l})$, para las cuatro concentraciones de alimento utilizadas.

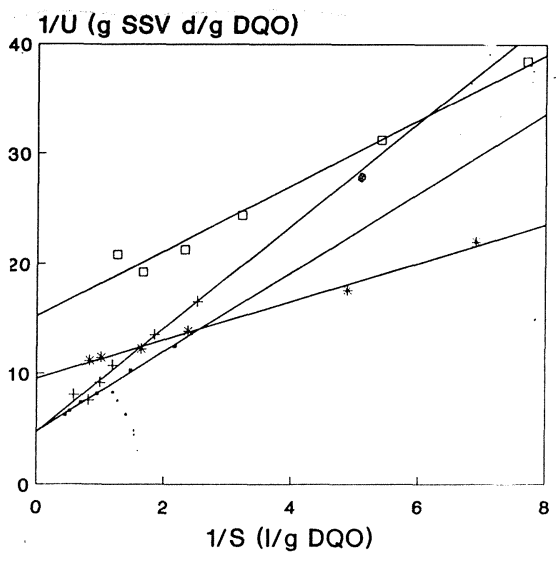

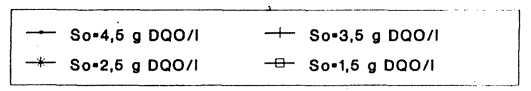

Figura 2

Representación de Lineweaver-Burk para la determinación de la velocidad máxima de utilización de sustrato $(k)$ y de la constante cinética del proceso $\left(K_{s}\right)$ para cada concentración de influente.

Por medio de la ecuación (4), y utilizando el método de regresión lineal de mínimos cuadrados, se han determinado los valores de los parámetros cinéticos $k$ y $K_{s}$ para cada concentración de sustrato utilizada como alimento (tabla II). Los coeficientes de regresión lineal obtenidos para las cuatro concentraciones de sustrato utilizadas como influente fueron: $0,998\left(S_{0}=4,5 \mathrm{~g} \mathrm{DQO} / \mathrm{l}\right), 0,988\left(S_{0}=3,5 \mathrm{~g} \mathrm{DQO} / \mathrm{l}\right)$, $0,997\left(S_{0}=2,5 \mathrm{~g} \mathrm{DQO} / \mathrm{l}\right)$ y $0,990\left(S_{0}=1,5 \mathrm{~g} \mathrm{DQO} / \mathrm{l}\right)$. Los elevados valores de los coeficientes de regresión, y en consecuencia, los pequeños límites de confianza de los parámetros cinéticos mencionados (tabla II) indican un buen grado de ajuste de los datos experimentales al modelo propuesto por Michaelis.

Tabla II

Valores de la velocidad máxima de utilización de sustrato, $k$ (g DQO/g SSV día), y de la constante cinética del proceso, $K_{\mathrm{s}}(\mathrm{g} \mathrm{DQO} / \mathrm{I})$, con sus límites de confianza $(P<0,05)$ para las distintas concentraciones de sustrato $S_{0}$ (g DQO/I) utilizadas

\begin{tabular}{lcccc}
\hline & $s_{0}=4,5 \mathrm{~g}$ DQO/ & $s_{0}=3,5 \mathrm{~g} \mathrm{DQO} /$ & $s_{0}=2,5 \mathrm{~g} \mathrm{DQO} /$ & $S_{0}=1,5 \mathrm{~g} \mathrm{DQO} /$ \\
\hline $\mathrm{k}$ (g DQO/g SSV d) & $0,22 \pm 0,01$ & $0,20 \pm 0,09$ & $0,10 \pm 0,01$ & $0,06 \pm 0,01$ \\
$\mathrm{~K}_{\mathbf{s}}(\mathrm{g} \mathrm{DQO} / \mathrm{l})$ & $0,76 \pm 0,12$ & $0,99 \pm 0,08$ & $0,18 \pm 0,03$ & $0,17 \pm 0,10$ \\
\hline
\end{tabular}

Como se observa en la tabla II, los parámetros cinéticos $k$ y $K_{\mathrm{s}}$ disminuyen al disminuir la concentración de sustrato en el alimento, siendo significativamente diferentes los valores correspondientes al alimento más concentrado $\left(S_{0}=4,5 \mathrm{~g} \mathrm{DQO} / \mathrm{l}\right)$ y más diluido $\left(S_{0}=1,5 \mathrm{~g} \mathrm{DQO} / \mathrm{l}\right)$. Shieh y colaboradores (1985) obtuvieron valores similares a los observados en este trabajo para concentraciones de sustrato en el alimento alta y media, utilizando sustratos sintéticos con glucosa como única fuente de carbono y carbón activado como soporte para la inmovilización de los microorganismos anaerobios responsables del proceso. Valores algo mayores se obtuvieron en el proceso de digestión anaerobia de aguas residuales de industrias cárnicas utilizando reactores de lecho fluidizado con bentonita como medio soporte (Borja et al., 1995b).

Por último, para la determinación de los coeficientes de rendimiento microbiano, $Y_{x}$, y muerte celular, $k_{\mathrm{d}}$, se utiliza el modelo de Lawrence y McCarty (1969), que viene dado por la ecuación:

$$
1 / \theta_{\mathrm{c}}=Y_{\mathrm{x}} U-k_{\mathrm{d}}
$$

siendo $\theta_{c}$ el tiempo de retención celular y $U$ la velocidad específica de utilización de sustrato.

El tiempo de retención celular se determina mediante la ecuación:

$$
\theta_{c}=\theta X / X_{e}
$$

donde $X$ y $X_{e}$ representan las concentraciones de biomasa ( $g$ SSV/l) en el reactor y efluente respectivamente.

De acuerdo con la ecuación (5), una representación del inverso del tiempo de retención celular frente a $U$ debe dar lugar a una línea recta de ordenada en el origen $-k_{d}$ y pendiente $Y_{x}$. En la figura 3 se representan las parejas de valores $\left(1 / \theta_{c}, U\right)$ para las cuatro concentraciones de sustrato estudiadas, 
observándose un buen ajuste lineal. Utilizando el procedimiento indicado y el método de regresión lineal de mínimos cuadrados se han obtenido los siguientes valores de los parámetros (coeficiente de regresión: 0,997):

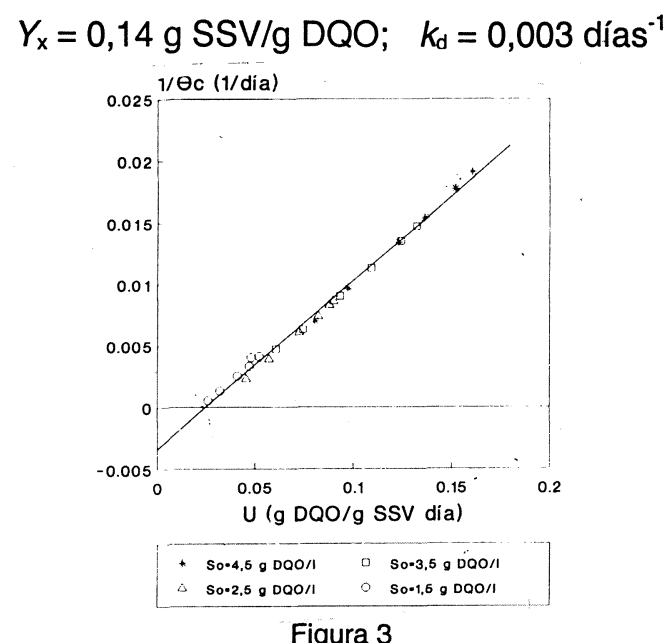

Variación de la velocidad específica de utilización de sustrato $U$, con el inverso del tiempo de retención celular $\left(1 / \theta_{c}\right)$.

Según Stronach et al., (1986), los valores máximos del coeficiente de rendimiento celular de las bacterias acetogénicas y metanogénicas son, respectivamente, 0,18 y $0,03 \mathrm{~g} \mathrm{SSV} / \mathrm{g} \mathrm{DQO}$. Considerando que en este trabajo se ha utilizado un cultivo mixto heterogéneo, resulta difícil evaluar la cinética de estas dos poblaciones microbianas separadamente. Sin embargo, un adecuado control de la fase metanogénica es clave para el óptimo comportamiento operacional del reactor, debido fundamentalmente al pequeño valor del coeficiente de producción celular observado en este tipo de bacterias en relación a los correspondientes valores de las acetogénicas. Valores similares del coeficiente de producción microbiana a los encontrados para la digestión anaerobia de aguas de lavado de aceitunas se obtuvieron en el tratamiento anaerobio de aguas residuales del proceso de elaboración de helados y derivados lácteos azucarados utilizando reactores de lecho de lodo granular, tipo UASB (upflow anaerobic sludge blanket) (Borja y Banks, 1994).

\section{CONCLUSIONES}

1.- Se ha comprobado que el modelo propuesto por Michaelis-Menten es aplicable al proceso continuo de digestión anaerobia de aguas de lavado de aceitunas de almazara en reactores de mezcla completa con biomasa inmovilizada en sepiolita.

2.- La velocidad máxima de utilización de sustrato $(k)$ y la constante cinética del proceso $\left(K_{s}\right)$ disminuyen al disminuir la concentración de sustrato en el alimento, presentando los valores más elevados ( $k=0,22 \mathrm{~g} \mathrm{DQO} / \mathrm{g}$ SSV día y $K_{\mathrm{s}}=0,76 \mathrm{~g} \mathrm{DQO} /$ ) para la concentración de alimento más alta estudiada $\left(S_{0}=4,5 \mathrm{~g} \mathrm{DQO} / \mathrm{l}\right)$, significativamente mayores que los obtenidos para la concentración de influente $\left(S_{0}=1,5 \mathrm{~g} D Q O / /\right)$ más baja utilizada $(k=0,06 \mathrm{~g}$ $\mathrm{DQO} / \mathrm{g}$ SSV día y $\left.K_{\mathrm{s}}=0,17 \mathrm{~g} \mathrm{DQO} / \mathrm{l}\right)$.

3.- Los coeficientes de rendimiento microbiano, $Y_{\mathrm{x}}$, y de muerte celular, $k_{\mathrm{d}}$, son: $0,14 \mathrm{~g} \mathrm{SSV} / \mathrm{g}$ DQO y 0,003 días $^{-1}$, respectivamente.

\section{AGRADECIMIENTOS}

Queremos hacer constar nuestro agradecimiento a la Unión Europea (Proyecto AIR3-CT94-1355) y a la Junta de Andalucía por su ayuda para la realización de estos estudios; y a $D .{ }^{a}$ Carmen Sánchez por su eficaz ayuda y colaboración en la parte experimental.

\section{BIBLIOGRAFÍA}

Alba, J. (1997).— «Elaboración de aceite de oliva virgen». En: «El cultivo del olivo». Ed. D. Barranco, D. Fernández y L. Rallo. Junta de Andalucía. Consejería de Agricultura y Pesca, 509-537.

American Public Health Association, APHA. (1985).- - «Standard Methods for the Examination of Water and Wastewater" (16th edn.).-APHA Washington, D.C.

Borja, R., Martín, A. y Fiestas, J.A. (1991)._«Influencia de la concentración de sustrato sobre la cinética del proceso de biometanización de alpechín en biorreactores de lecho fluidizado".-Grasas y Aceites 42, 363-370.

Borja, R., Alba, J. e Hidalgo, F. (1994)._- ¿Digestión anaerobia de las aguas de lavado de aceitunas de almazara: influencia del período de recolección sobre la cinética del proceso".-Grasas y Aceites 45, 126-131.

Borja, R. and Banks, C.J. (1994).-- «Kinetics of an upflow anaerobic sludge blanket reactor treating ice-cream wastewater".-Environmental Technology 15, 219-232.

Borja, R., Banks, C.J., Alba, J. and Escobar, J.P. (1995a). - "The temperature dependence of the kinetic parameters derived for the aerobic treatment of wastewater from the washing of olives prior to the oil production process".-J. Environ. Sci. Health A30, 8, 1693-1705.

Borja, R., Banks, C.J. and Wang, Z. (1995b).- "Kinetic evaluation of an anaerobic fluidised-bed reactor treating slaughterhouse wastewater".-Bioresource Technology 52, 163-167.

Borja, R., Alba, J., Martín, A. y Mancha, A. (1998)."Influencia de la velocidad de carga orgánica sobre el proceso de depuración anaerobia de aguas de lavado de aceitunas de almazara en reactores de lecho fluidizado".-Grasas y Aceites 49, 42-49.

Fannin; K.F. (1987).- «Start-up, operation, stability and control». En: "Anaerobic digestion of biomass".Eds.: D.P. Chynoweth and R. Isaacson, Elsevier Applied Science, 171-196. 
Field, J., Sierra-Alvarez, R. and Lettinga, G. (1988)."Ensayos anaerobios".-4 $4^{\circ}$ Seminario de Digestión Anaerobia de Aguas Residuales. Secretariado de Publicaciones de la Universidad de Valladolid.

Fiestas, J.A. and Borja, R. (1996)._-Biomethanization».International Biodeterioration and Biodegradation 38, 3-4, 145-153.

Guiot, S.R., Podruzny, M.F. and McLean, D.D. (1989)."Assessment of macroenergetic parameters for an anaerobic upflow biomass bed and filter (UBF) reactor".-Biotechnol. Bioeng. 34, 1277-1288.

Lawrence, A.W. and Stover, E.L. (1969)._-Kinetics of methane fermentation in anaerobic treatment".- J. Water Pollut. Control Fed., 41, R1-R16.

Maestrojuán, G.M. and Fiestas, J.A. (1988).— «A study of behaviour of anaerobic bacteria in the presence of diverse materials usable as support".-Proceedings of $\mathrm{V}$ International Symposium on Anaerobic Digestion, Bologna, Italy, 129-132.
Olthof, M. and Oleszkiewick, J. (1982).- «Anaerobic treatment of industrial wastewaters".-Chemical Engineering, 15, 121-126.

Shieh, W.K., Chun, T.L. and Chen, S.J. (1985).«Performance evaluation of the anaerobic fluidized-bed system: III. Process kinetics".-J. Chem. Tech. Biotechnol., 35B, 229-234.

Stronach, S.M., Rudd, T. and Lester, J.N. (1986)."Anaerobic digestion processes in industrial wastewater treatment".-Springer-Verlag, Berlin.

Water Pollution Control Federation (W.P.C.F.). (1967)."Anaerobic sludge digestion. Manual of practise".n. ${ }^{\circ} 16$. 